\title{
Training Simulator for Acquiring Operational Skill to Operate Overhead Traveling Crane while Suppressing Load Sway
}

\author{
Yoshiyuki Noda $\left(\mathbb{D},{ }^{1}\right.$ Ryuki Hoshi, ${ }^{1}$ and Akihiro Kaneshige $\mathbb{i D}^{2}$ \\ ${ }^{1}$ Course of Mechanical Engineering, Integrated Graduate School of Medicine, Engineering, and Agricultural Sciences, \\ University of Yamanashi, Kofu 400-8511, Japan \\ ${ }^{2}$ Department of Mechanical Engineering, Toyota College, National Institute of Technology, Toyota 471-8525, Japan \\ Correspondence should be addressed to Yoshiyuki Noda; noday@yamanashi.ac.jp
}

Received 14 February 2019; Revised 5 April 2019; Accepted 18 April 2019; Published 13 May 2019

Academic Editor: Stefano Marchesiello

Copyright (c) 2019 Yoshiyuki Noda et al. This is an open access article distributed under the Creative Commons Attribution License, which permits unrestricted use, distribution, and reproduction in any medium, provided the original work is properly cited.

\begin{abstract}
The present study addressed an advanced system that was designed to teach the skills needed to operate an electric overhead traveling crane. In the operation of an overhead traveling crane, to ensure the safe and efficient transport of a load, an operator must be able to suppress the sway of that load. However, considerable training is needed to master this skill. Furthermore, the beginners may cause accidents by operating the crane incorrectly. Therefore, in the present study, we devised a training system in which an operator can safely and efficiently master the operation of an electric overhead traveling crane while suppressing the load sway. In this approach, the operational conditions in which the load sway is suppressed can be derived theoretically. The operational conditions are displayed by the crane simulator as visual instructions in a virtual working environment. The efficacy of the proposed skill training approach with the crane simulator was verified through experiments whereby novices used the crane simulator to perform the task of transporting a load while suppressing load sway.
\end{abstract}

\section{Introduction}

Electric overhead traveling cranes are commonly used for transporting materials and products in manufacturing industry as shown in Figure 1. Using these cranes, suspended loads can be transported efficiently in three-dimensional space. However, the load is prone to swaying due to suspending mechanism. This load swaying disturbs the precise positioning, and there is a risk of its collision with other objects on the factory floor [1-3]. Therefore, in the operation of an overhead traveling crane, one of the basic skills is the suppression of load sway. However, mastering this skill requires considerable hands-on practice. Furthermore, there is the danger of accidents occurring when a novice is operating the crane. Moreover, the characteristics of pendanttype operating devices with button-type momentary switches, which are commonly found on overhead traveling cranes, make it even more difficult to suppress the load sway, given that the momentary switches limit the motions of the crane's moving parts to ON/OFF operations. Therefore, there is a demand for a means of safely and efficiently acquiring the skills needed to suppress load sway in an overhead traveling crane operated by a device with momentary switches.

The studies of the load sway control system in the overhead traveling crane are commonly reported in the literature. The dynamics and the control strategies of the overhead traveling crane have been reviewed by some researchers $[1,4,5]$. The feedback control systems for suppressing the load sway have been proposed using the statevariable feedback control [6], the adaptive control [7], the root locus method $[2,8]$, the fuzzy control [9-11], the nonlinear PID control based on Lyapunov stability theorem [12], the error tracking control law for arbitrary initial load sway angle [13], and the dual-model matching method [14]. Furthermore, the auxiliary effectors installed to the crane hook or the cart have been developed for suppressing the load sway [15-17]. The feedforward control systems taking 


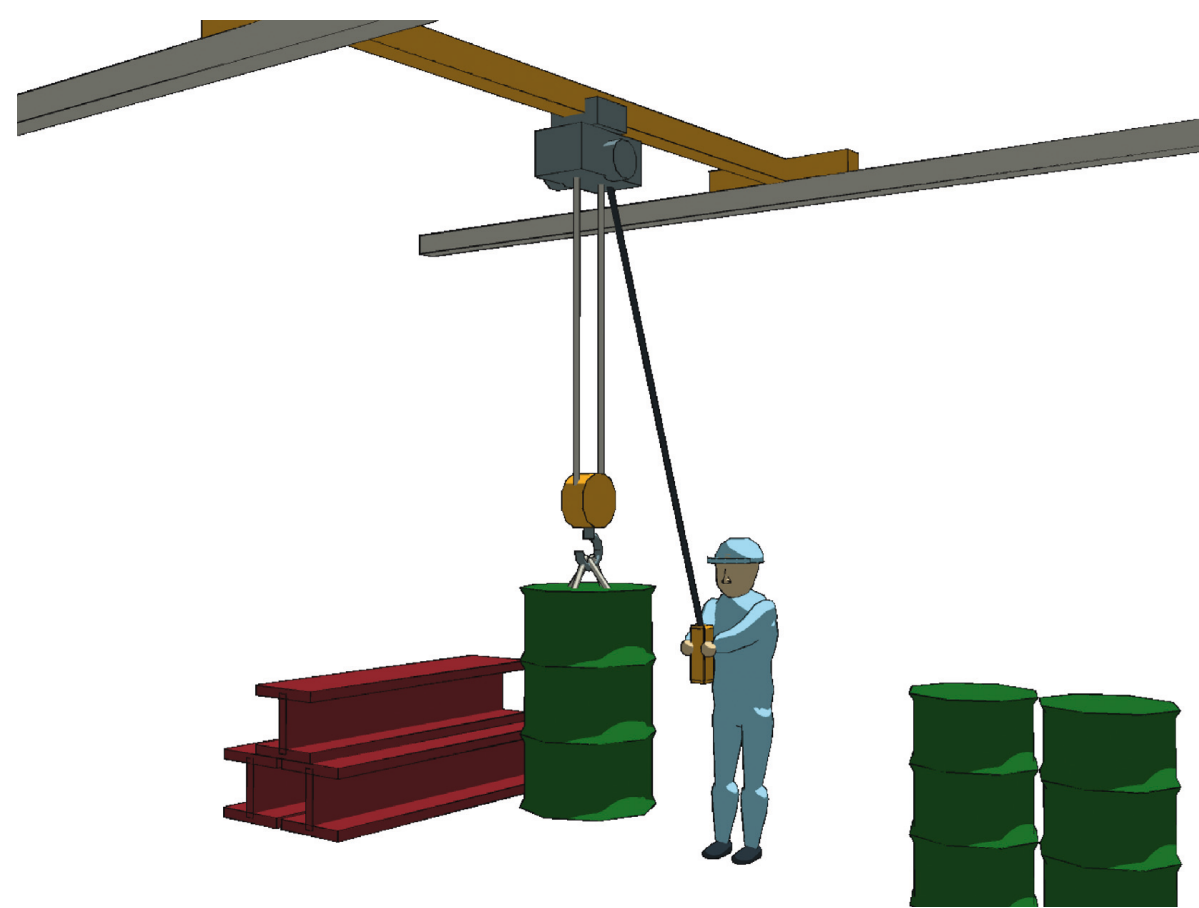

FIGURE 1: Illustration of electric overhead traveling crane with pendant-type operating device.

into account the load sway suppression have been proposed using the optimal control approach [18], the input shaper [19], the trajectory planning by neural networks [20], and the notch filter [21]. Many researchers have dealt with the problems with regard to the load sway suppression in the overhead traveling crane.

Recently, some technologies for improving crane operation have been developed. An operational assistance system using a haptic device has been developed for suppressing load sway with a rough-terrain crane [22]. This device is installed in a joystick which is used to operate the crane, and an assistance force for suppressing the load sway is provided. To safely operate such a crane on the likes of a construction site, a crane operation assistance system using augmented reality technology has been proposed [23]. Through this approach, a transport path that would avoid collisions with other objects is indicated on the displayed image. Furthermore, an operational assistance system using a joystick with a force display has been proposed as a means of suppressing load sway and avoiding obstacles when operating an overhead traveling crane [24]. With any assistance systems, however, training is required to realize the effective use of the assistance technologies [25].

Training simulators for a crane system have been developed $[26,27]$. These have introduced multiscreen displays [26] and structure-motion joint simulation [27] to improve the practicality of the crane operating environment. Previous studies $[28,29]$ set out to develop a simulator using a force displayed joystick for providing training in the operation of a shipboard crane. Therefore, a trainee can learn how to operate the crane while suppressing the load sway based on the reaction force display and the visual information provided by the simulator. However, most conventional studies addressing crane training systems have assumed the use of a joystick. To the best of our knowledge, the training system for the operation of a crane while suppressing the load sway, using momentary switches, has not been reported before. Furthermore, there has been little discussion on how to efficiently acquire the crane operating skills while suppressing the load sway using a visual crane simulator.

Therefore, a training system by which a trainee can efficiently and safely acquire the skills needed to operate an electric overhead traveling crane using a momentary switch while suppressing the load sway is proposed in this paper. The operational conditions for suppressing the load sway are derived theoretically. Then, instructions for suppressing the load sway are displayed in the training mode in the crane simulator. The trainee can efficiently and safely acquire the operational skills with the training simulator using the proposed training approach.

The remainder of this paper is organized as follows. Section 2 provides an overview of a training system whereby the crane simulator is operated by momentary switches. The operational conditions for suppressing the load sway are derived theoretically in Section 3. Then, the method for displaying the operational conditions is described in Section 4. The efficacy of the proposed training system is verified through experiments with subjects, as described in Section 5. Finally, we summarize our findings.

\section{Operational Training Simulator for Overhead Traveling Crane}

In the present study, an operational training simulator for an overhead traveling crane was constructed as shown in Figure 2. The virtual working environment for the overhead traveling crane and the instructions for operating the crane 


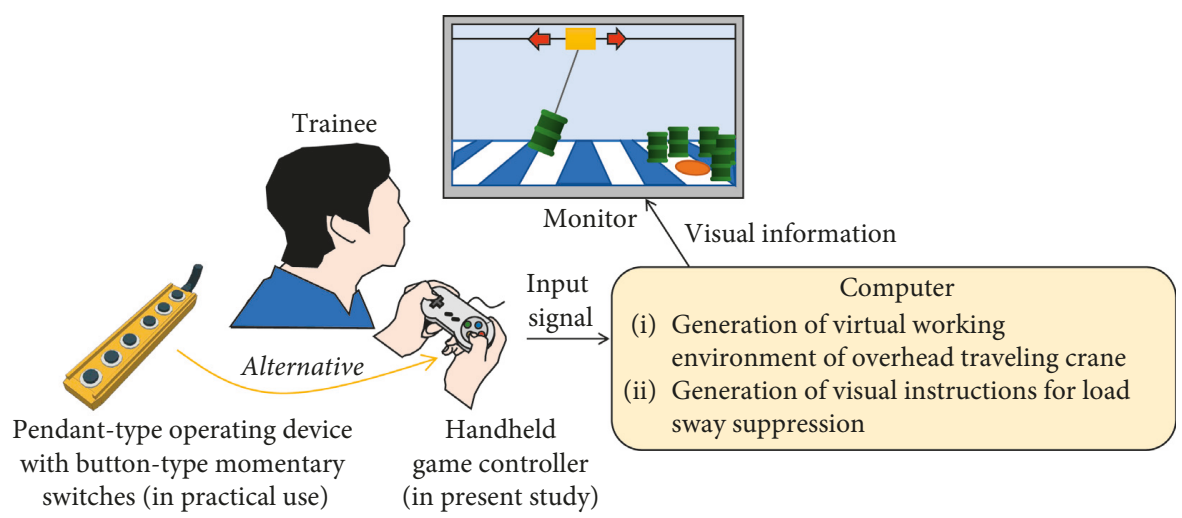

FIgURE 2: Overview of the operational training simulator of overhead traveling crane.

while suppressing the load sway are generated by the computer and displayed on the monitor. The trainees can operate the virtual overhead traveling crane using the operational device. A pendant-type operating device with momentary switches is typically used to operate an overhead traveling crane. However, in the present study, a handheld game controller with momentary switches was used to facilitate the construction of the crane simulator. The virtual working environment of the overhead traveling crane is programmed in Unity, which is commonly used for the creation of computer games. The cart from which the load is suspended can be transported laterally in the virtual environment. The load sways due to the movement of this cart.

The virtual working environment of the overhead traveling crane was constructed using the crane model shown in Figure 3(a). The crane model can be represented as

$$
\begin{aligned}
\ddot{x} & =u, \\
m l^{2} \ddot{\theta} & =-m g l \sin \theta+m \ddot{x} l \cos \theta,
\end{aligned}
$$

where $x$ is the amount of travel of the cart and $u$ is the input signal for controlling the cart motion. $\theta$ is the angle of the rope caused by the swaying of the load. $m, l$, and $g$ are the mass of the load, rope length given as a length from the cart to a gravity center of the load, and acceleration due to gravity, respectively. In the present study, it was assumed that the cart moves accurately in response to the input signal. Therefore, the transport acceleration of the cart is equal to the input signal, as given by Equation (1). Furthermore, since the load sway in the model does not consider the viscosity or friction, it is represented as an undamped vibration model, as defined in Equation (2).

In an overhead traveling crane, the cart continues to move in one direction provided that the momentary switch on the operating device is held down. By pressing or releasing a momentary switch, the input signal $u$ is generated as shown in Figure 3(b). Therefore, the input signal can be represented as

$$
u= \begin{cases}a, & \left(t_{p} \leq t<t_{p}+T_{a}\right), \\ -a, & \left(t_{r} \leq t<t_{r}+T_{a}\right), \\ 0, & (\text { else }),\end{cases}
$$

where $a$ is the amplitude of the transport acceleration and $T_{a}$ is the acceleration time. $t_{p}$ and $t_{r}$ are the instants at which the switch is pressed and released, respectively. As shown in Figure 3(b), the cart is transported with a trapezoidal velocity curve pattern with a constant velocity, $v=a T_{a}$.

\section{Crane Operation with Load Sway Suppression}

This section proposes a method whereby an overhead traveling crane can be operated with momentary switches, while suppressing the load sway. Since the motion of the cart in a crane operated by momentary switches is limited as shown in Figure 3(b), it is difficult to apply common vibration suppression approaches such as the notch filter method $[21,30]$. Therefore, the input shaper approach, whereby the vibration is suppressed through the application of multiple signal pulses, has been proposed $[19,31,32]$. This approach is a type of feedforward vibration suppression control, where the vibration caused by the original signal can be suppressed by the countervibration caused by the phase lag signal.

We developed an operational method that would suppress load sway in a crane controlled with momentary switches by using an input shaper approach. In a conventional input shaper, the input signal is controlled automatically with time management. However, it is difficult to accurately apply this time management when a crane is being operated by a human. Therefore, we propose an operational method based on the angle information of the load sway. First, a load sway suppression method when an impulse signal is used as the input command is derived as the basic concept. Subsequently, an operational method that suppresses the load sway is proposed for the crane, which is operated using the momentary switches. Finally, we analyse the robustness of the proposed operational method for variations in the rope length.

3.1. Load Sway Suppression when Using an Impulse Signal. The application of load sway suppression when using an impulse signal is shown in Figure 4. In a crane operated by momentary switches, the cart is accelerated when the momentary switch is pressed and decelerated when the momentary switch is released, as shown in Figure 3(b). The 


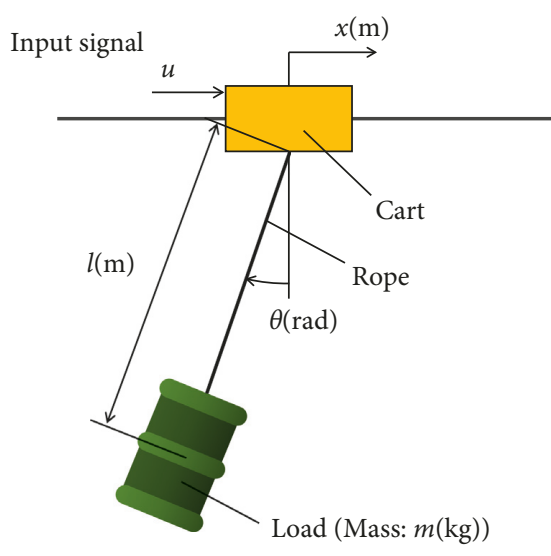

(a)

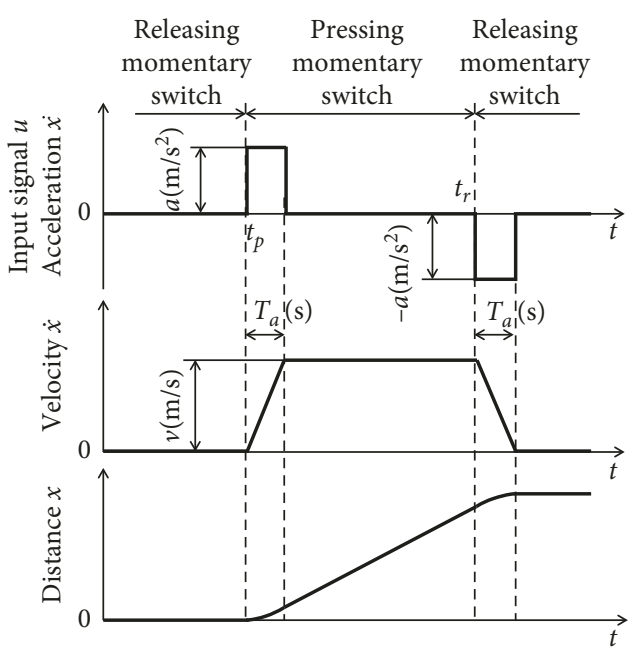

(b)

Figure 3: Overview of the overhead traveling crane model with operating momentary switches. (a) Illustration of crane model. (b) Cart motion operated by momentary switch.

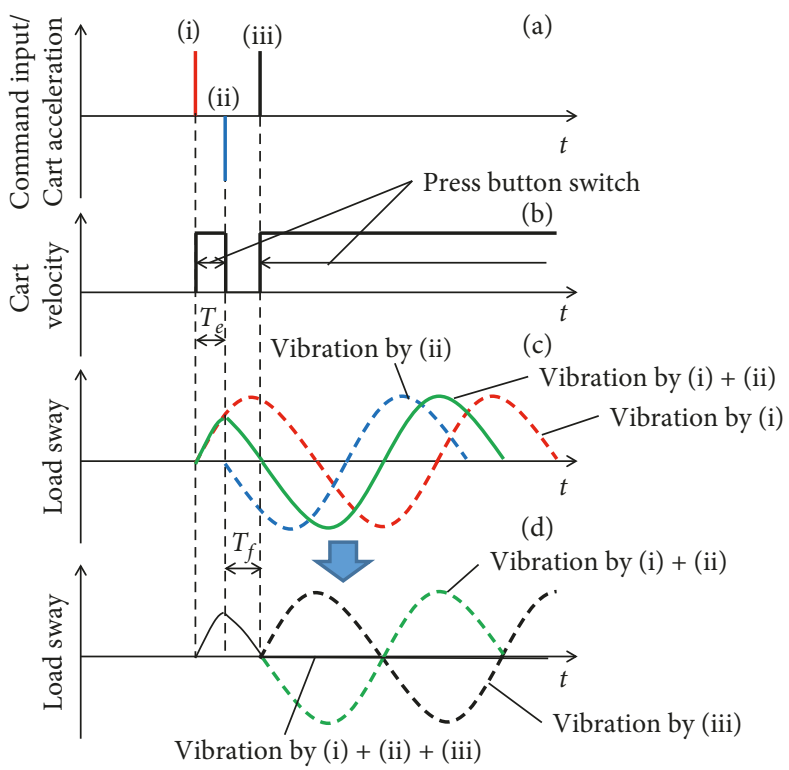

FIGURE 4: Overview of proposed operation for suppressing load sway when using an impulse command input. (a) Command input regarding as the cart acceleration with (i) acceleration generated by pressing the momentary switch, (ii) deceleration generated by releasing the momentary switch, and (iii) acceleration generated by re-pressing the momentary switch. (b) Cart velocity. (c) Load sway occurred by the cart accelerations (i) and (ii). (d) Load sway occurred by the cart accelerations (i), (ii), and (ii).

amplitudes of the acceleration and deceleration are equal. In Figure 4(c), load sway, indicated by the red broken line occurs due to the acceleration (i). After $T_{e} \mathrm{~s}$, the load sway indicated by the blue broken line occurs due to the deceleration (ii). Here, $T_{e}$ can be derived by superimposing the vibrations caused by (i) and (ii), with the amplitude being the same as that of the vibration caused by a single impulse signal. Therefore, the vibration caused by the superposition of the vibrations resulting from (i) and (ii) can be suppressed by the countervibration produced by the acceleration (iii). $T_{f}$ is the time span between the deceleration (ii) and the acceleration (iii). $T_{e}$ and $T_{f}$ can be derived analytically as follows.
In the design of the load sway suppression method, the load sway model defined by Equation (2) is linearized with $\theta \ll 1$ as

$$
m l^{2} \ddot{\theta}=-m g l \theta+m l u
$$

When the unit impulse signal used as the command input $u$ is added to the crane system at $t=0$, the angle of the load sway can be derived as

$$
\theta_{1}(t)=A \sin (\omega t)
$$

where the natural angular frequency and the amplitude of the load sway can be represented by $\omega=\sqrt{g / l}$ and 
$A=1 / \sqrt{g l}$, respectively. Equation (5) represents the angle $\theta_{1}$ of the load sway that occurs as a result of the impulse acceleration (i) shown in Figure 4. Therefore, the load sway angles $\theta_{2}$ and $\theta_{3}$ occur due to the impulse deceleration (ii) and acceleration (iii), respectively, and can be represented as

$$
\begin{aligned}
& \theta_{2}(t)=-A \sin \left(\omega t-\omega T_{e}\right)=-A \sin \left(\omega t-\phi_{e}\right), \\
& \theta_{3}(t)=A \sin \left\{\omega t-\omega\left(T_{e}+T_{f}\right)\right\}=A \sin \left(\omega t-\phi_{e}-\phi_{f}\right),
\end{aligned}
$$

where the initial phases for the angles $\theta_{2}$ and $\theta_{3}$ can be shown as $\phi_{e}=\omega T_{e}$ and $\phi_{f}=\omega T_{f}$, respectively. The load sway resulting from the superposition of the vibrations caused by (i) and (ii) can be derived as

$$
\begin{aligned}
\theta_{1+2}(t) & =\theta_{1}(t)+\theta_{2}(t)=A \sin (\omega t)-A \sin \left(\omega t-\phi_{e}\right) \\
& =A \sin \left(\omega t+\phi_{s}\right)
\end{aligned}
$$

Here, the harmonic vibration can be obtained by the superposition of two harmonic vibrations [33] as

$$
C \sin (\omega t+\phi)=C_{1} \sin \left(\omega t+\phi_{1}\right)+C_{2} \sin \left(\omega t+\phi_{2}\right) .
$$

In Equation (9), the amplitudes and initial phases of the vibrations are related as

$$
\begin{aligned}
C & =\sqrt{C_{1}^{2}+C_{2}^{2}+2 C_{1} C_{2} \cos \left(\phi_{1}-\phi_{2}\right)}, \\
\tan \phi & =\frac{C_{1} \sin \phi_{1}+C_{2} \sin \phi_{2}}{C_{1} \cos \phi_{1}+C_{2} \cos \phi_{2}} .
\end{aligned}
$$

From Equation (10), the amplitudes of the vibrations given by Equation (7) are related as

$$
A=\sqrt{A^{2}+A^{2}-2 A^{2} \cos \phi_{e}}=A \sqrt{2\left(1-\cos \phi_{e}\right)} .
$$

The following equation can be derived to satisfy Equation (12).

$$
1-\cos \phi_{e}=\frac{1}{2}
$$

Therefore, the initial phase $\phi_{e}$ can be obtained as

$$
\phi_{e}=\frac{\pi}{3}
$$

and the interval $T_{e}$ also can be represented as

$$
T_{e}=\frac{\phi_{e}}{\omega}=\frac{\pi}{3 \omega} \text {. }
$$

By substituting $t=T_{e}$ into Equation (5), the condition under which the momentary switch should be released is given as

$$
\left.\theta_{1}(t)\right|_{t=T_{e}}=A \sin \left(\omega T_{e}\right)=A \sin \phi_{e}=\frac{\sqrt{3} A}{2} .
$$

The amplitude of the superposed vibration resulting from the accelerations (i) and (ii) can be made equal to that of vibration resulting from the acceleration (i) by releasing the momentary switch when the vibration is $\sqrt{3} / 2$ times amplitude $A$.
Then, the initial phase $\phi_{\mathrm{s}}$ of the superposed vibration can be derived using Equation (11) as

$$
\tan \phi_{\mathrm{s}}=\frac{-A \sin \left(-\phi_{e}\right)}{A-A \cos \left(-\phi_{e}\right)}=\frac{\sqrt{3}}{1} .
$$

Thus, the initial phase $\phi_{\mathrm{s}}$ of the superposed vibration is given as

$$
\phi_{\mathrm{s}}=\frac{\pi}{3}
$$

Next, the vibration resulting from the accelerations (i), (ii), and (iii) can be suppressed as shown in Figure 4(d) and therefore represented as

$$
\theta_{1+2+3}(t)=A \sin \left(\omega t+\phi_{s}\right)+A \sin \left(\omega t-\phi_{e}-\phi_{f}\right)=0 .
$$

Equation (19) can be rewritten with $t_{e}=t-T_{e}$ as

$$
\theta_{1+2+3}\left(t_{e}\right)=A \sin \left(\omega t_{e}+\phi_{s}+\phi_{e}\right)+A \sin \left(\omega t_{e}-\phi_{f}\right)=0 \text {. }
$$

The relation between the amplitudes in Equation (20) can be shown using Equation (10) as

$$
\begin{aligned}
0 & =\sqrt{A^{2}+A^{2}+2 A^{2} \cos \left(\phi_{s}+\phi_{e}+\phi_{f}\right)} \\
& =A \sqrt{2\left\{1+\cos \left(\phi_{s}+\phi_{e}+\phi_{f}\right)\right\}} .
\end{aligned}
$$

Thus, Equation (21) can be satisfied by the initial phase as

$$
\phi_{f}=\frac{\pi}{3}
$$

and the interval $T_{f}$ also can be represented as

$$
T_{f}=\frac{\phi_{f}}{\omega}=\frac{\pi}{3 \omega} \text {. }
$$

By substituting $t_{e}=T_{f}$ into Equation (8), the condition for re-pressing the momentary switch can be clarified as

$$
\begin{aligned}
\left.\theta_{1+2}\left(t_{e}\right)\right|_{t_{e}=T_{f}} & =\left.A \sin \left(\omega t_{e}+\phi_{s}+\phi_{e}\right)\right|_{t_{e}=T_{f}} \\
& =A \sin \left(\phi_{f}+\phi_{s}+\phi_{e}\right)=A \sin \pi=0 .
\end{aligned}
$$

The superposed vibration by the accelerations (i), (ii), and (iii) can be suppressed by re-pressing the momentary switch when the superposed vibration by the accelerations (i) and (ii) is 0 .

3.2. Load Sway Suppression when Operating Crane Using a Momentary Switch. As described in Section 2, the cart is transported according to a trapezoidal curve pattern. Therefore, the operation for suppressing the load sway, described in Section 3.1, should be extended to apply in the trapezoidal curve pattern generated by Equation (3).

A Laplace transform of the load sway model is shown as

$$
\Theta(s)=\frac{1}{\sqrt{g l}} \frac{\omega_{n}}{s^{2}+\omega^{2}}\left(\frac{a}{s}-e^{-s T_{a}} \frac{a}{s}\right),
$$

where $s$ is the Laplace operator. Equation (25) can be derived by substituting the trapezoidal curve pattern before deceleration 
into the load sway model defined by Equation (4). The inverse Laplace transform to Equation (25) can be obtained as

$$
\theta(t)=\frac{a}{g} \sqrt{2-2 \cos \omega T_{a}} \sin \left(\omega t-\frac{\omega T_{a}}{2}\right) .
$$

By comparing Equations (26) and (5), it can be clarified that the response of the load sway with the trapezoidal curve pattern lags $\left(\omega T_{a}\right) / 2 \mathrm{~s}$ behind that with the impulse signal. Therefore, the condition for releasing the momentary switch in the case of the trapezoidal curve pattern can be clarified as

$$
\begin{aligned}
\left.\theta_{1}(t)\right|_{t=T_{e}-T_{a} / 2} & =A_{t} \sin \omega\left(T_{e}-\frac{T_{a}}{2}\right)=A_{t} \sin \left(\phi_{e}-\frac{\omega T_{a}}{2}\right) \\
& =A_{t} \sin \left(\frac{\pi}{3}-\frac{\omega T_{a}}{2}\right),
\end{aligned}
$$

where the amplitude of the load sway is given by $A_{t}=$ $a / g \sqrt{2-2 \cos \omega T_{a}}$. Furthermore, the condition for repressing the momentary switch in the case of the trapezoidal curve pattern can also be represented as

$$
\begin{aligned}
\left.\theta_{1+2}\left(t_{e}\right)\right|_{t_{e}=T_{f}-T_{a} / 2} & =A_{t} \sin \left(\phi_{f}+\phi_{s}+\phi_{e}-\frac{\omega T_{a}}{2}\right) \\
& =A_{t} \sin \left(\pi-\frac{\omega T_{a}}{2}\right) .
\end{aligned}
$$

The load sway that occurs after starting the motion of the cart can be suppressed by operating the momentary switch under the sway angle conditions given by Equations (27) and (28). Furthermore, the load sway that occurs after the cart has stopped after moving to the desired position can also be suppressed by operating the momentary switch with the sway angle conditions by alternately releasing and then repressing the switch.

\subsection{Efficacy of Proposed Approach to Load Sway Suppression} for Varying Rope Lengths. When a crane is actually being used, the rope length can be varied to suit the environment and the object being lifted. It is possible to suppress the load sway by applying the operating method of the momentary switches described in Section 3.2, although the rope length is varied. The efficacy of the proposed approach for suppressing load sway despite the varying rope length is verified mathematically in this subsection.

The necessary conditions for operating the momentary switch to suppress the load sway are given by Equations (27) and (28) can be for different rope lengths as follows:

$$
\begin{array}{r}
\theta_{i}=\frac{a}{g} \sqrt{2-2 \cos \left(\omega_{\mathrm{st}}+\delta_{\omega}\right) T_{a}} \sin \left(\phi_{i}-\frac{\left(\omega_{\mathrm{st}}+\delta_{\omega}\right) T_{a}}{2}\right) \\
i=e, h,
\end{array}
$$

where $i=e$, and $i=h$ indicate the sway angle conditions when the momentary switch is released and re-pressed, respectively. Therefore, $\phi_{e}=\pi / 3$ and $\phi_{h}=\pi$. The operator aims to attain the operational conditions for suppressing the load sway with the standard natural angular frequency $\omega_{\text {st }}$, which is represented for a standard rope length $l_{\text {st }}$ as $\omega_{\text {st }}=\sqrt{g / l_{\text {st }}} \cdot \delta_{\omega}$ is the error between the standard and practical natural angular frequencies, caused by the differences in the rope lengths. Equation (29) can be represented as

$$
\begin{aligned}
\theta_{i}= & \frac{2 a}{g} \sin \frac{\omega_{\mathrm{st}} T_{a}+\delta_{\omega} T_{a}}{2} \sin \left(\phi_{i}-\frac{\omega_{\mathrm{st}} T_{a}+\delta_{\omega} T_{a}}{2}\right) \\
= & \frac{2 a}{g}\left(\sin \frac{\omega_{\mathrm{st}} T_{a}}{2} \cos \frac{\delta_{\omega} T_{a}}{2}+\cos \frac{\omega_{\mathrm{st}} T_{a}}{2} \sin \frac{\delta_{\omega} T_{a}}{2}\right) \\
& \cdot\left\{\sin \left(\phi_{i}-\frac{\omega_{\mathrm{st}} T_{a}}{2}\right) \cos \frac{\delta_{\omega} T_{a}}{2}-\cos \left(\phi_{i}-\frac{\omega_{\mathrm{st}} T_{a}}{2}\right) \sin \frac{\delta_{\omega} T_{a}}{2}\right\} .
\end{aligned}
$$

We assume that $\delta_{\omega} T_{a} / 2$ is small and can be approximated as $\sin \left(\delta_{\omega} T_{a} / 2\right) \approx \delta_{\omega} T_{a} / 2, \quad \cos \left(\delta_{\omega} T_{a} / 2\right) \approx 1$, and $\left(\delta_{\omega} T_{a} / 2\right)^{2} \approx 0$. By substituting these approximations into Equation (30), the operational conditions can be given as

$$
\theta_{i}=\frac{2 a}{g}\left\{\sin \frac{\omega_{\mathrm{st}} T_{a}}{2} \sin \left(\phi_{i}-\frac{\omega_{\mathrm{st}} T_{a}}{2}\right)+\frac{\delta_{\omega} T_{a}}{2} \sin \left(\phi_{i}-\omega_{\mathrm{st}} T_{a}\right)\right\} .
$$

As defined by Equation (31), if $\left|\delta_{\omega} T_{a} / 2\right| \ll 1$, the load sway with the error between the standard and practical natural frequencies can be suppressed by the operational conditions derived from the standard natural angular frequency.

The numerical verification of the above analysis is given by the following example. The standard rope length is $l_{\mathrm{st}}=3 \mathrm{~m}$, and the practical rope length is $l_{\mathrm{pr}}=2 \mathrm{~m}$. Therefore, the standard and practical natural angular frequencies are $\omega_{\mathrm{st}}=1.81 \mathrm{rad} / \mathrm{s}$ and $\omega_{\mathrm{pr}}=2.21 \mathrm{rad} / \mathrm{s}$, respectively. The error in the natural angular frequency is $\delta_{\omega}=-0.4 \mathrm{rad} / \mathrm{s}$. We give the acceleration time as $T_{a}=0.3 \mathrm{~s}$. Thus, $\left|\delta_{\omega} T_{a} / 2\right|=0.06 \ll 1$. Upon releasing the momentary switch, the first term in the curly bracket in Equation (31) is calculated as $0.188 \mathrm{rad}(10.7 \mathrm{deg})$. Conversely, the second term is given as $-0.029 \mathrm{rad}(-1.7 \mathrm{deg})$. Therefore, any error between the standard and practical natural angular frequencies has a relatively minor effect on the operational conditions. The condition $\left|\delta_{\omega} T_{a} / 2\right| \ll 1$ can be realized with a small error $\delta_{\omega}$ in the natural angular frequency and/or a short acceleration time $T_{a}$.

3.4. Verification of Proposed Approach by Numerical Simulations. The efficacy of the proposed approach for suppressing load sway was verified by the numerical simulations. With the proposed approach, the momentary switch is operated according to the sway angle, as defined by Equations (27) and (28). The sway angles are derived from the standard rope length, $l_{\mathrm{st}}=3 \mathrm{~m}$, the acceleration time, $T_{a}=0.3 \mathrm{~s}$, and the amplitude of acceleration, $a=5 \mathrm{~m} / \mathrm{s}^{2}$. Therefore, the sway angle at which the momentary switch is released and then re-pressed can be derived as $\theta_{1}=0.191$ 
$\operatorname{rad}(11.0 \mathrm{deg})$ and $\theta_{1+2}=0.073 \mathrm{rad}(4.2 \mathrm{deg})$, respectively. In the simulation, the rope length $l_{\text {sim }}$ is the same as the standard rope length $l_{\text {st. }}$. The simulation results are shown in Figure 5. In Figures 5(a)-5(d) show the acceleration, velocity, position of the cart, and load sway angle, respectively. As shown in Figure 5(d), the load sway can be suppressed positively by the proposed approach.

Furthermore, it was clarified that the proposed approach is an effective means of suppressing the load sway, although the rope length differs from the standard length. For comparison, the momentary switch was operated at the timings introduced in the conventional approaches $[20,28]$. The standard rope length, acceleration time, and amplitude of the acceleration are the same as in the previous simulation. Therefore, the sway angles in the proposed approach are the same as those used in the previous simulation. The timings at which the momentary switch is released and re-pressed in the conventional approach, as shown in Figure 4 , can be derived as $T_{e}=T_{f}=$ $\pi /\left(3 \omega_{\mathrm{st}}\right)=0.58 \mathrm{~s}$. In the simulations, the rope length is $l_{\text {sim }}=2 \mathrm{~m}$. Therefore, the error between the standard and simulation natural angular frequencies is $\delta_{\omega}=-0.4 \mathrm{rad} / \mathrm{s}$. The simulation results are shown in Figure 6, which is presented in the same way as the graph in Figure 5. The blue and red lines show the simulation results obtained with the proposed and conventional approaches, respectively. In the operation based on timings, since the input signal applied to the cart motion is designed for a feedforward control approach, it is difficult to suppress the load sway error between the standard and simulation natural angular frequencies. Conversely, since the error between the standard and simulation natural angular frequencies has little effect on the proposed approach, as described in Section 3.3, the load sway caused by an error in the natural frequency can be suppressed by operating according to the sway angle conditions in the proposed approach as shown in Figure 6.

\section{Display of Operational Conditions for Load Sway Suppression in Crane Simulator}

The operational skills required to suppress load sway can be acquired by training the operation of a momentary switch using the overhead traveling crane simulator. With the simulator, the sway angles proposed in the previous section are displayed in the virtual working environment, as shown in Figure 7. A drum, acting as the load, is suspended from the cart. When the cart is transported on the right side, the sway angles at which the momentary switch are released and repressed are indicated as the leftmost and middle translucent drums in the monitor, respectively. The leftmost translucent drum is displayed in red until the momentary switch is released. Furthermore, the middle translucent drum is displayed in blue from the instant that the momentary switch is released until it is re-pressed. Thus, the operator can efficiently master the skills needed to suppress the load sway by observing the translucent drums at the different sway angles.

\section{Experimental Verification Involving Use of Crane Simulator}

The efficacy of the proposed approach was verified by experiments using the crane simulator. The experimental procedure for a single subject is shown in Figure 8(a). At the beginning of the experiments, the operator makes five attempts to move the cart while suppressing the load sway, for a rope length of $4 \mathrm{~m}$. The operation trials were performed without displaying the operational instructions as shown in Figure 8(b). After the trials, the operator made 20 training runs, performing the operations necessary to suppress the load sway. Then, the operator made another five attempts to operate the cart while suppressing the load sway. Finally, five attempts were undertaken with the rope length set to $5 \mathrm{~m}$. The training for operators comprised learning the necessary skills in one of the following three training environments:

(i) The sway angle conditions being displayed by the simulator, as shown in Figure 7.

(ii) No operational instructions for suppressing the load sway are displayed. The operator was forced to determine the best method for suppressing the load sway.

(iii) To memorize the timings, the operator practiced the operation according to the time-series graph shown in Figure 9. This approach was proposed in [28].

The subjects of the experiments were 12 males in their early twenties. They are mechanical engineering students in the author's university. We received informed consent from all study subjects. In the questionnaire before the experiments, they answered that they were inexperienced at operating an overhead traveling crane and have no knowledge of the operation method for suppressing the load sway. Each subject individually performed the experiments in line with the procedure as shown in Figure 8(a) without the other subjects seeing. Four of the subjects trained the simulator, which displayed the sway angle conditions. Four other subjects trained without any guidance on how to suppress the load sway. The remaining four subjects trained using the time-series graph. The subjects were randomly assigned to the three groups.

The experimental results for one of those subjects who trained with the sway angle conditions displayed are shown in Figure 10. Figures $10(\mathrm{a})-10$ (c) show the experimental results obtained from the 5th attempt before training, the 1st attempt after training, and the 1st attempt after changing the rope length to $5 \mathrm{~m}$, respectively. Furthermore, the cart accelerations, cart velocities, and the angles of the load sway are shown in the upper, middle, and lower graphs, respectively. As shown in Figure 10(a), the operator does not know the best means of suppressing the load sway and cannot determine how to operate the crane, even after five trials. An inexperienced subject finds it difficult to suppress the load sway. After the training with displaying the sway angle conditions, the operator can acquire the skills needed to suppress the load sway, as shown in Figure 10(b). 


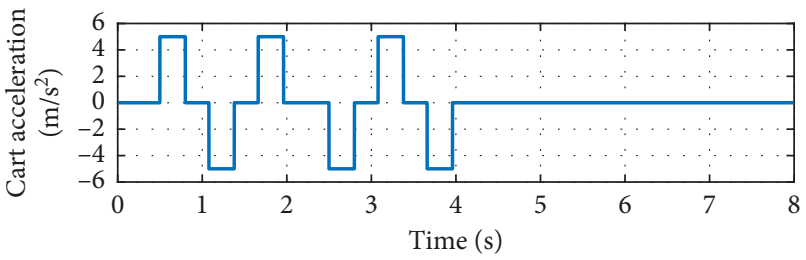

(a)

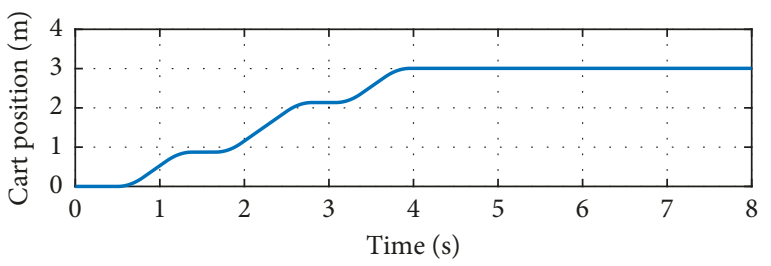

(c)

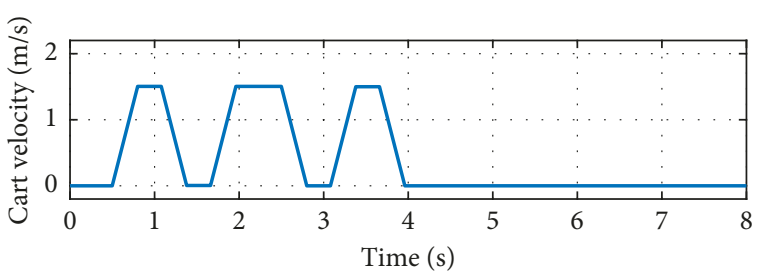

(b)

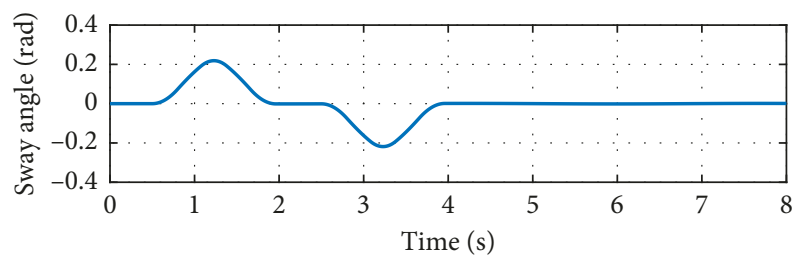

(d)

FIGURE 5: Simulation results of cart motion and load sway in overhead traveling crane with the proposed operational method, which has no error between standard and simulated rope lengths as $l_{\mathrm{st}}=l_{\text {sim }}=3 \mathrm{~m}$. (a) Cart acceleration. (b) Cart velocity. (c) Cart position. (d) Angle of load sway.

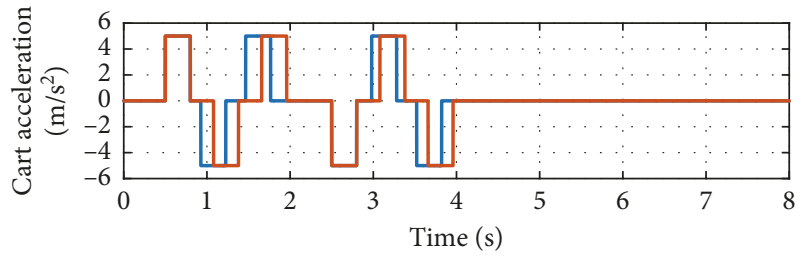

- Sway angle conditions _ Timings

(a)

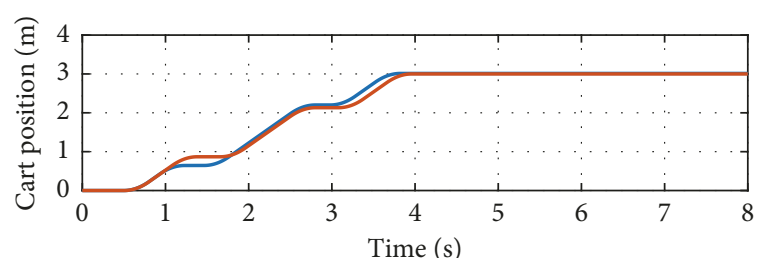

- Sway angle conditions - Timings

(c)

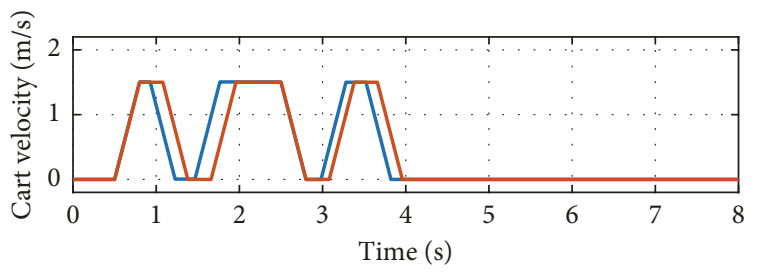

- Sway angle conditions __ Timings

(b)

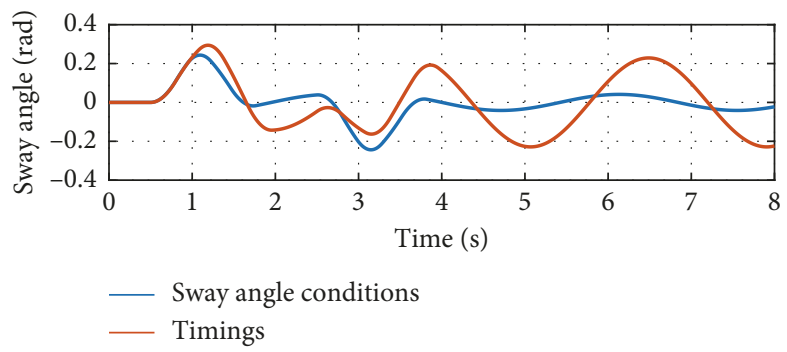

(d)

FIGURE 6: Simulation results of cart motion and load sway in overhead traveling crane with proposed and conventional operational methods, which have error between standard and practical rope lengths as $l_{\text {st }}=3 \mathrm{~m}$ and $l_{\text {sim }}=2 \mathrm{~m}$. (a) Cart acceleration. (b) Cart velocity. (c) Cart position. (d) Angle of load sway. The blue lines represent the simulation results by operating the momentary switch based on sway angle conditions proposed in this study. The red lines represent the simulation results by operating the momentary switch based on timings reported in the conventional approach.

Moreover, the load sway can still be suppressed even if the rope length is changed from 4 to $5 \mathrm{~m}$, as shown in Figure $10(\mathrm{c})$. The error in the natural angular frequency upon changing the rope length from 4 to $5 \mathrm{~m}$ is $\delta_{\omega}=0.165 \mathrm{rad} / \mathrm{s}$. The acceleration time of cart is $T_{a}=0.3 \mathrm{~s}$. Therefore, since $\left|\delta_{\omega} T_{a} / 2\right|=0.025 \ll 1$, as derived in Section 3.3 , the effect of changing the rope length is minimal on suppression of the load sway.

The amplitudes of the residual load sways (i.e., the load sway after the cart has been moved to its final position) attained by each operator in the attempts are shown in
Figure 11. The mean amplitude of the residual load sway is obtained by taking the average of the amplitudes of the residual load sways attained in five attempts before the training, after the training, and after changing the rope length. Figures 11(a)-11(c) show the results attained by the operators trained by displaying the sway angle conditions, without any instructions for suppressing the load sway, and with the time-series graph, respectively. The markers indicating the mean amplitudes are connected by the lines with respect to the subjects. Figure 11(b) shows that when no instructions are provided, suppressing the load sway is 


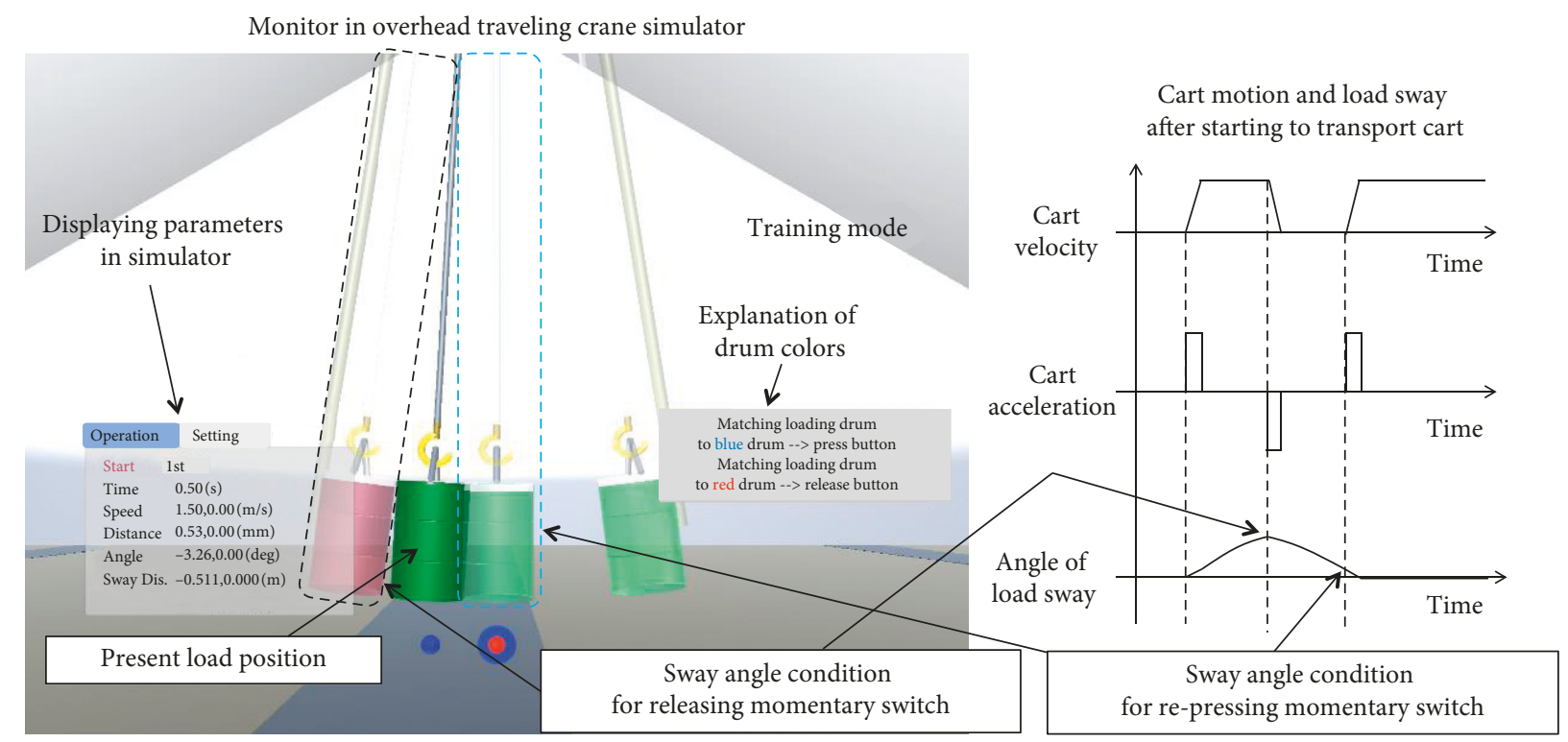

FIgURE 7: Monitor display of sway angles in overhead traveling crane simulator.

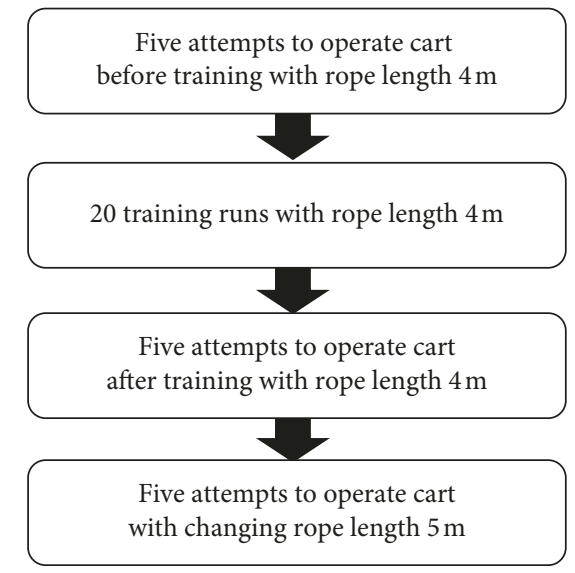

(a)

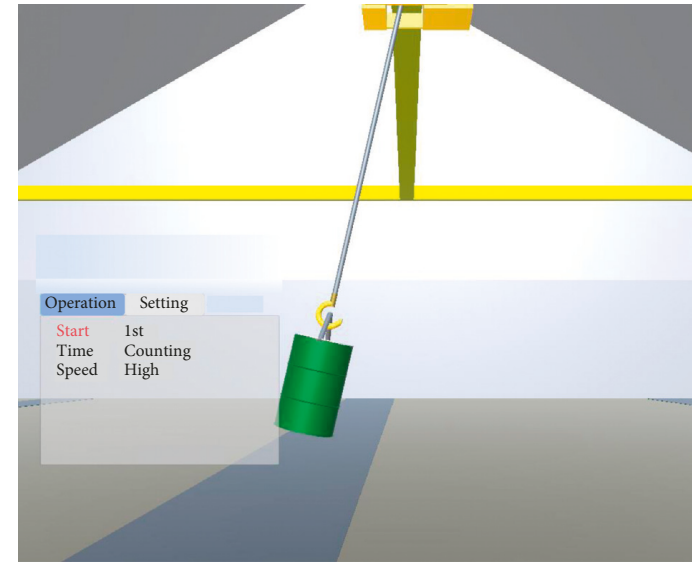

(b)

Figure 8: Procedure of experiments and monitor display in attempts. (a) Procedure of experiments for a single subject. (b) Monitor display in attempts in which the operational instructions are hidden.

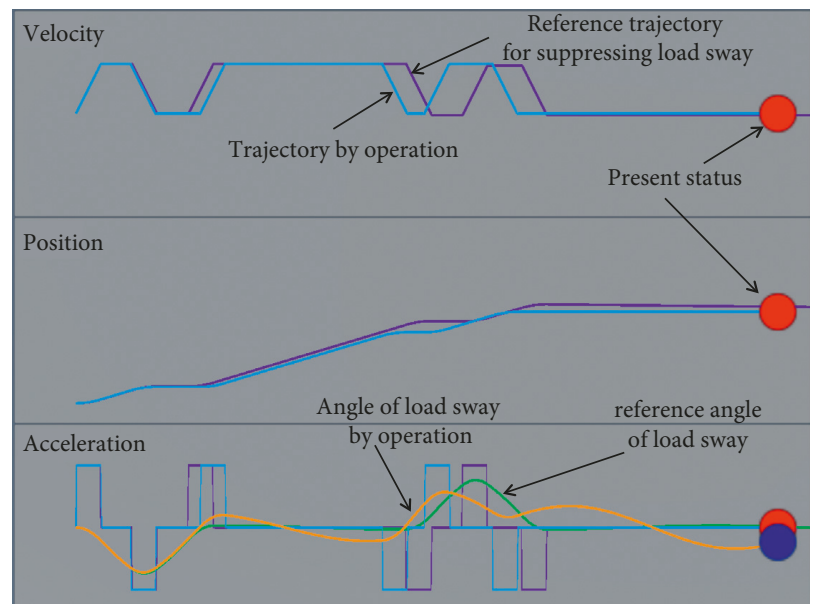

FIGURE 9: Monitor display with time-series graph in training for memorizing timings of operation. 

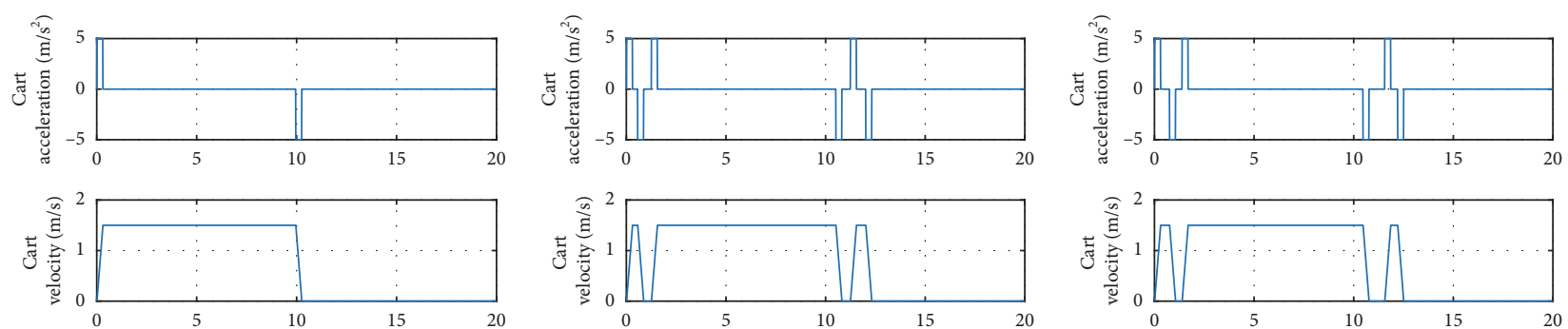

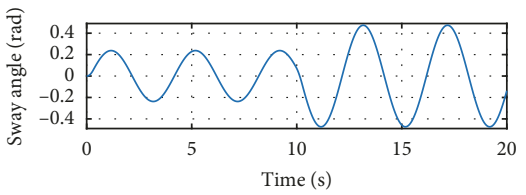

(a)

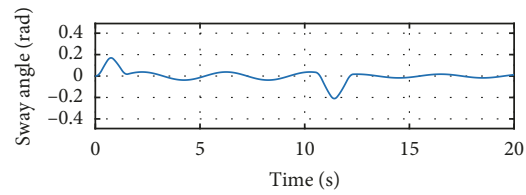

(b)

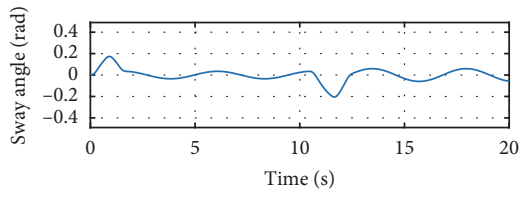

(c)

Figure 10: Experimental results which subject A-1 attempts to operate the cart in case of training with sway angles displayed in the simulator. (a) The experimental results in 5th attempt before training. (b) The experimental results in 1st attempt after training. (c) The experimental results in 1st attempt after changing to rope length $5 \mathrm{~m}$.

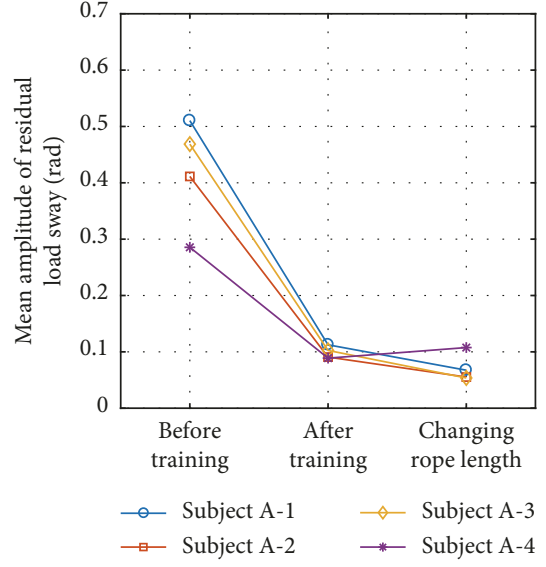

(a)

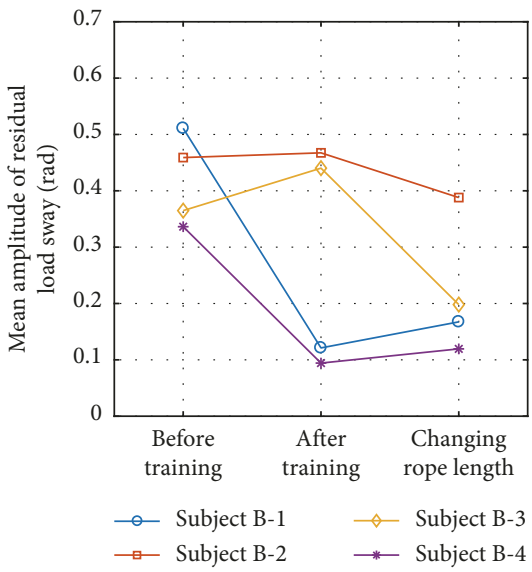

(b)

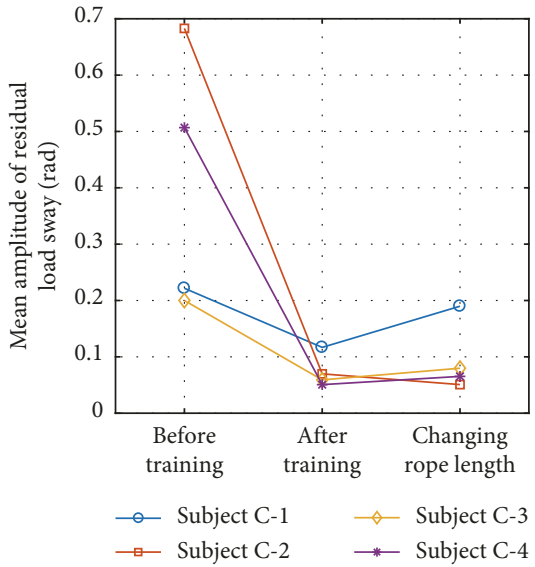

(c)

Figure 11: Mean amplitudes of residual load sway in attempts. (a) The results of which the subjects train the crane operations with displaying the sway angle conditions shown in Figure 7. (b) The results of which the subjects train the crane operations without displaying operational instructions. (c) The results of which the subjects train the crane operation with displaying the time-series graph shown in Figure 9. The results of each subject are associated with the colored line.

difficult even after training 20 times. In contrast, operators who trained with the displayed sway angles could suppress the load sway as shown in Figure 11(a). Furthermore, most operators who had been trained with the displayed sway angle could suppress the load sway better with a rope length $5 \mathrm{~m}$ than a rope length $4 \mathrm{~m}$. This is attributed to the improvement of the operator's skills by increasing the number of trials and suppressing the fluctuations in the operations by increasing the time period of the load sway. The operators who trained using the time-series graph could also suppress the load sway. However, the variations in the amplitude of the residual load sway were greater than those for the operators who had trained with the sway angle displayed. During the training with the time-series graph, the operators memorized the rhythm with the timings. After the trials, however, they remarked that it was difficult to accurately maintain the rhythm while operating the crane. Furthermore, since the load sway resulting from change in the rope length cannot be suppressed by the operations based on a memorized rhythm, the operators were forced to newly determine the best way of suppressing the load sway.

From the results of the experiments with the simulator, it can be said that the approach, whereby the operator trains with the sway angles displayed, is the most effective means of acquiring the skills needed to operate the momentary switches in a way that suppresses the load sway.

\section{Conclusions}

In the present study, we devised an innovative operational skill training system for suppressing the load sway inherent to an overhead traveling crane by using a crane simulator. The following conclusions can be drawn: 
(1) By developing an overhead traveling crane simulator operated by momentary switches, a beginner trainee can safely learn the skills needed to suppress load sway

(2) The operation of the momentary switches for suppressing the load sway was analysed theoretically, and we proposed an operating method based on the sway angles

(3) Operation based on the sway angles is an effective means of suppressing the load sway, with the error between the standard and practical rope lengths considered under the condition $\left|\delta_{\omega} T_{a} / 2\right| \ll 1$

(4) In the experiments using the crane simulator, the operator could effectively acquire the operational skills required to suppress the load sway by training with the sway angles displayed by the crane simulator

The present study used only a crane simulator. In our future work, the efficacy of the proposed approach will be verified by using an actual overhead traveling crane operated by momentary switches.

\section{Data Availability}

The data used to support the findings of this study are available from the corresponding author upon request.

\section{Conflicts of Interest}

The authors declare that there are no conflicts of interest regarding the publication of this paper.

\section{Acknowledgments}

A part of this study was supported by JKA Foundation (No. 2018M-177).

\section{References}

[1] E. M. Abdel-Rahman, A. H. Nayfeh, and Z. N. Masoud, "Dynamics and control of cranes: a review," Modal Analysis, vol. 9, no. 7, pp. 863-908, 2003.

[2] H.-H. Lee, "Modeling and control of a three-dimensional overhead crane," Journal of Dynamic Systems, Measurement, and Control, vol. 120, no. 4, pp. 471-476, 1998.

[3] A. Belunce, V. Pandolfo, H. Roozbahani, and H. Handroos, "Novel control method for overhead crane's load stability," Procedia Engineering, vol. 106, pp. 108-125, 2015.

[4] L. Ramli, Z. Mohamed, A. M. Abdullahi, H. I. Jaafar, and I. M. Lazim, "Control strategies for crane systems: a comprehensive review," Mechanical Systems and Signal Processing, vol. 95, pp. 1-23, 2017.

[5] K.-S. Hong and U. H. Shah, Dynamics and Control of Industrial Cranes, Advanced in Industrial Control, Springer, Singapore, 2019.

[6] H. T. Nguyen, "State-variable feedback controller for an overhead crane," Journal of Electrical and Electronics Engineering, vol. 14, no. 2, pp. 75-84, 1994.

[7] F. Boustany and B. d'Andrea-Novel, "Adaptive control of an overhead crane using dynamic feedback linearization and estimation design," in Proceedings of the 1992 IEEE
International Conference on Robotics and Automation, pp. 1963-1968, Nice, France, May 1992.

[8] H.-H. Lee, S.-K. Cho, and J.-S. Cho, "A new anti-swing control of overhead cranes," IFAC Proceedings Volumes, vol. 30, no. 13, pp. 115-120, 1997.

[9] J. Yi, N. Yubazaki, and K. Hirota, "Anti-swing fuzzy control of overhead traveling crane," in Proceedings of the IEEE International Conference on Fuzzy Systems, vol. 2, pp. 12981303, Honolulu, Hawaii, May 2002.

[10] J. Yi, N. Yubazaki, and K. Hirota, "Anti-swing and positioning control of overhead traveling crane," Information Sciences, vol. 155, no. 1-2, pp. 19-42, 2003.

[11] M. Z. Othman, "A new approach for controlling overhead traveling crane using rough controller," International Journal of Computer and Information Engineering, vol. 2, no. 2, pp. 581-585, 2008.

[12] H.-H. Lee, "A new approach for the anti-swing control of overhead cranes with high-speed load hoisting," International Journal of Control, vol. 76, no. 15, pp. 1493-1499, 2003.

[13] M. Zhang, X. Ma, X. Rong, X. Tian, and Y. Li, "Error tracking control for underactuated overhead cranes against arbitrary initial payload swing angles," Mechanical Systems and Signal Processing, vol. 84, pp. 268-285, 2017.

[14] Y. Mori and Y. Tagawa, "Vibration controller for overhead cranes considering limited horizontal acceleration," Control Engineering Practice, vol. 81, pp. 256-263, 2018.

[15] O. Sawodny, H. Aschemann, and S. Lahres, "An automated gantry crane as a large workspace robot," Control Engineering Practice, vol. 10, no. 12, pp. 1323-1338, 2002.

[16] H. Aschemann, "Passivity-based control of an overhead traveling crane," in Proceedings of the 17th World Congress the International Federation of Automatic Control, pp. 76787683, Seoul, Korea, July 2008.

[17] H. Osumi, M. Kubo, S. Yano, and K. Saito, "Development of tele-operation system for a crane without overshoot in positioning," in Proceedings of the IEEE/RSJ International Conference on Intelligent Robots and Systems, pp. 5799-5805, Taipei, Taiwan, October 2010.

[18] A. Z. Al-Garni, K. A. F. Moustafa, and S. S. A. K. Javeed Nizami, "Optimal control of overhead cranes," Control Engineering Practice, vol. 3, no. 9, pp. 1277-1284, 1995.

[19] J. Vaughan, E. Maleki, and W. Singhose, "Advantages of using command shaping over feedback for crane control," in Proceedings of the 2010 American Control Conference, pp. 2308-2313, Baltimore, MA, USA, July 2010.

[20] A. Abe, "Anti-sway control for overhead cranes using neural networks," International Journal of Innovative Computing, Information and Control, vol. 7, no. 7, pp. 4251-4262, 2011.

[21] A. Kaneshige, Y. Kawasaki, S. Ueki, and S. Nagai, "Development of an autonomous mobile overhead traveling crane with on-line obstacle recognition and path-planning based on obstacle information-the design of a transfer control system in consideration of oscillating control," in Proceedings of the 2nd International Symposium on Computer, Communication, Control and Automation, pp. 468-473, Singapore, December 2013.

[22] M. Yoneda, F. Arai, T. Fukuda, K. Miyata, and T. Naito, "Assistance system for crane operation with haptic displayoperational assistance to suppress round payload swing," in Proceedings of the 1999 IEEE International Conference on Robotics and Automation, pp. 2924-2929, Detroit, MI, USA, May 1999.

[23] Yi-C. Chen, H.-L. Chi, S.-C. Kangm, and S.-H. Hsieh, "A smart crane operations assistance system using augmented 
reality technology," in Proceedings of the 28th International Symposium for Automation and Robotics in Construction, pp. 643-649, Seoul, Korea, June 2011.

[24] Y. Hara and Y. Noda, "Operational assistance system for obstacle collision avoidance and load sway suppression in overhead traveling crane," in Proceedings of the 2016 IEEE International Conference on Systems, Man, and Cybernetics, pp. 2196-2201, Budapest, Hungary, 2016.

[25] T. Kuneguchi, Y. Noda, Y. Sago, and K. Kakihara, "Operational assistance system using 3-DOF joystick with reaction force display to load transfer machine in a plane," in Proceedings of the 11th International Conference on Informatics in Control, Automation and Robotics, pp. 346-352, Vienna, Austria, September 2014.

[26] Z. Lu, D. Chen, H. Dong, C. Shen, and Z. Liu, "Research on distributed multi-screen overhead crane simulator," in Proceedings of the Third International Conference on Pervasive Computing and Applications, pp. 1003-1006, Kunming, China, May 2008.

[27] K.-l. Lu, Y.-f. Huang, W. Yan, J. Hu, C. Chen, and D.-f. Chang, "Joint simulation of trolley vehicle-frame structure coupled vibration using ADAMS and ANSYS for container crane simulated training system," International Journal of Hybrid Information Technology, vol. 6, no. 5, pp. 197-208, 2013.

[28] N. Yong Jian, Y. Noda, and K. Terashima, "Simulator building for agile control design of shipboard crane and its application to operational training," IFAC Proceedings Volumes, vol. 44, no. 1, pp. 7375-7383, 2011.

[29] Y. Matsuda, S. Fushimi, and K. Terashima, "Development of training simulator for sway suppression skills on shipboard rotary cranes," in Proceedings of the 11th International Conference on Informatics in Control, Automation and Robotics, vol. 1, pp. 433-440, Vienna, Austria, September 2014.

[30] A. D. Smith, "Comparison of filtering methods for crane vibration reduction," Tower, vol. 1, no. 2, pp. 1-7, 2009.

[31] N. C. Singer and W. P. Seering, "Preshaping command inputs to reduce system vibration," Journal of Dynamic Systems, Measurement, and Control, vol. 112, no. 1, pp. 76-82, 1990.

[32] J. Vaughan, A. Yano, and W. Singhose, "Robust negative input shapers for vibration suppression," Journal of Dynamic Systems, Measurement, and Control, vol. 131, no. 3, p. 031014, 2009.

[33] D. Hartog, Mechanical Vibration, McGraw-Hill Book Company, New York, NY, USA, 1956. 


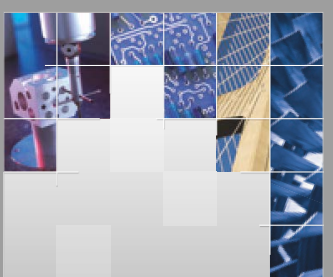

\section{Enfincering}
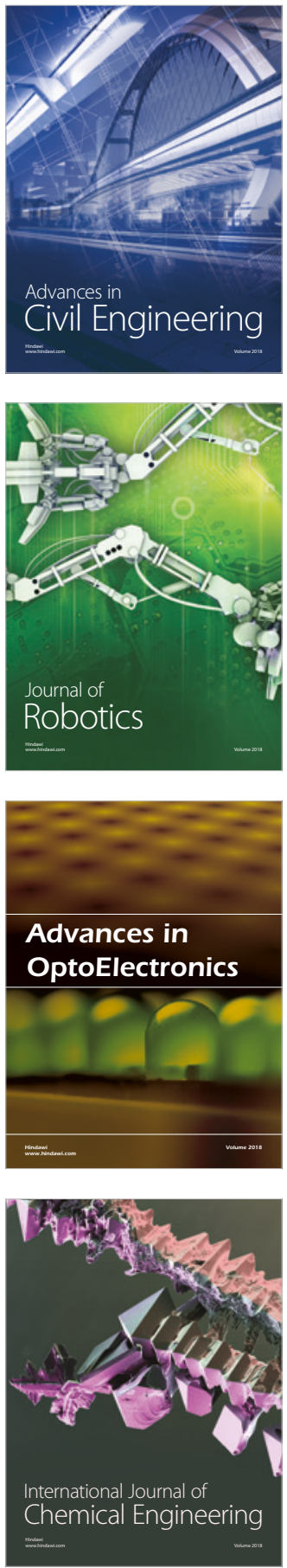

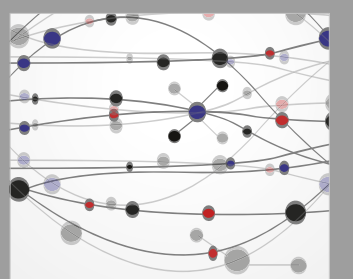

\section{Rotating \\ Machinery}

The Scientific World Journal

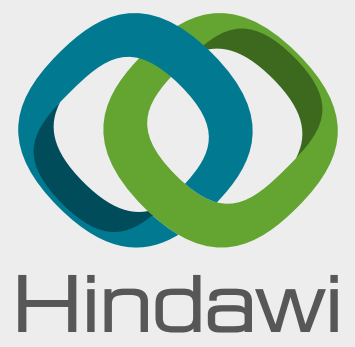

Submit your manuscripts at

www.hindawi.com
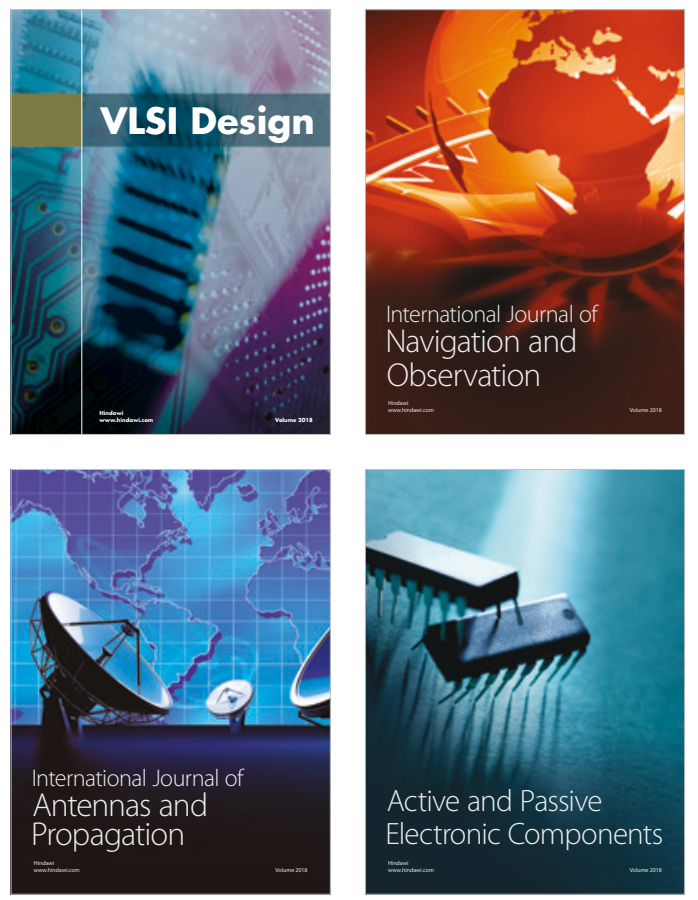
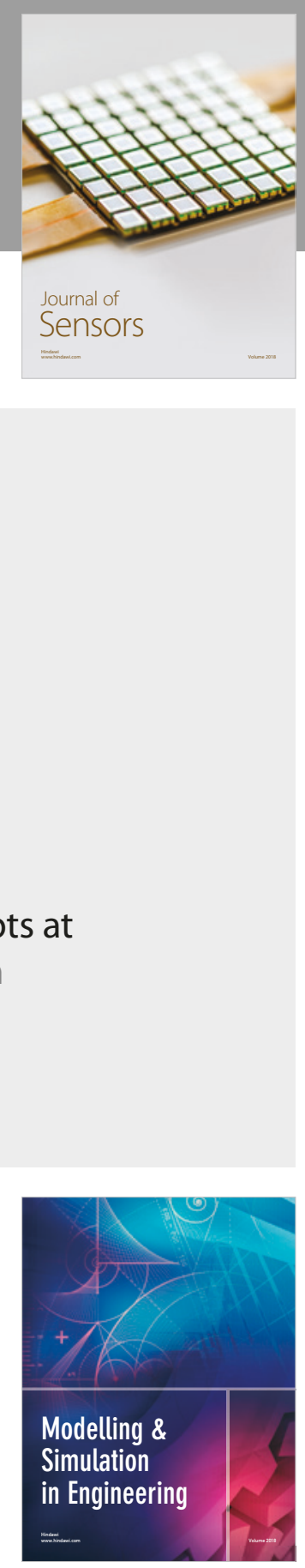

\section{Advances \\ Multimedia}
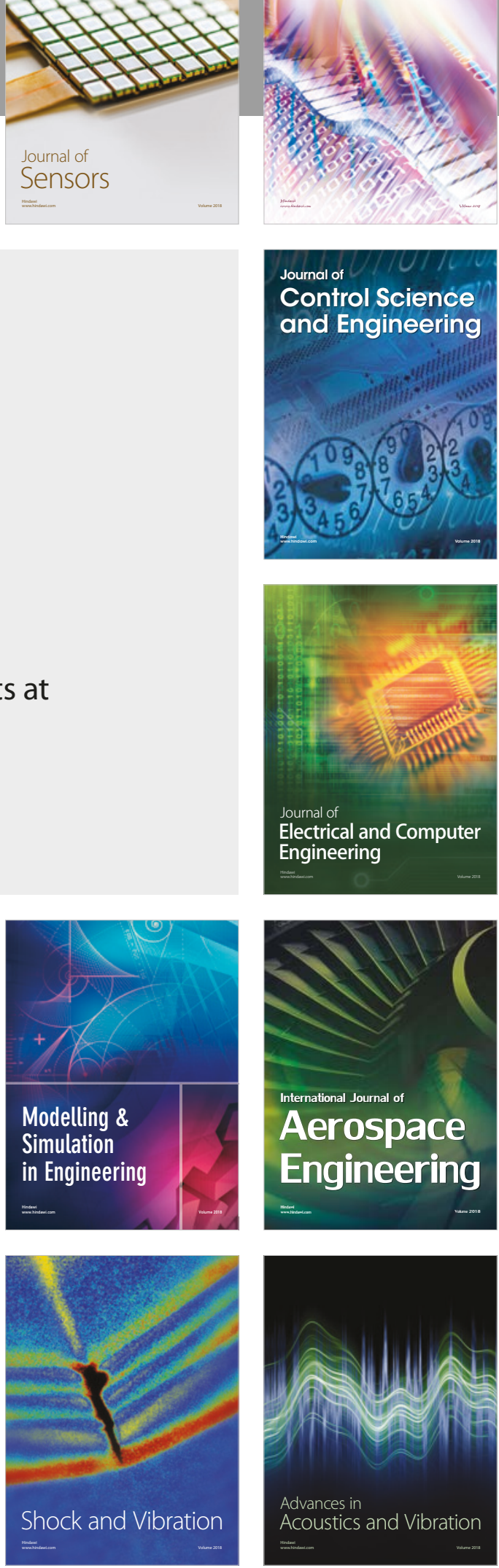